\title{
Loxapine to control agitation during weaning from mechanical ventilation
}

\author{
Stéphane Gaudry ${ }^{1,2}$, Benjamin Sztrymf ${ }^{3,4}$, Romain Sonneville ${ }^{5 \dagger}$, Bruno Megarbane ${ }^{6 \dagger}$, Guillaume Van Der Meersch, \\ Dominique Vodovar ${ }^{6}$, Yves Cohen ${ }^{7}$, Jean-Damien Ricard ${ }^{1,8}$, David Hajage ${ }^{2,9}$, Laurence Salomon ${ }^{9}$ \\ and Didier Dreyfuss ${ }^{1,8,10^{*}}$
}

\begin{abstract}
Background: Weaning from mechanical ventilation (MV) may be impeded by the occurrence of agitation. Loxapine has the ability to control agitation without affecting spontaneous ventilation. The aim of this study was to establish whether loxapine would reduce MV weaning duration in agitated patients.

Methods: We performed a multicentre, double-blind, placebo-controlled, parallel group, randomised trial. Patients who were potential candidates for weaning but exhibited agitation (Richmond Agitation-Sedation Scale score $\geq 2$ ) after sedation withdrawal were randomly assigned to receive either loxapine or placebo. In case of severe agitation, conventional sedation was immediately resumed. The primary endpoint was the time between first administration of loxapine or placebo and successful extubation.

Results: The trial was discontinued after 102 patients were enrolled because of an insufficient inclusion rate. Median times to successful extubation were 3.2 days in the loxapine group and 5 days in the placebo group (relative risk $1.2,95 \% \mathrm{Cl} 0.75-1.88, p=0.45)$. During the first $24 \mathrm{~h}$, sedation was more frequently resumed in the placebo group ( $44 \%$ vs $17 \%, p=0.01$ ).

Conclusions: In this prematurely stopped trial, loxapine did not significantly shorten weaning from MV. However, loxapine reduced the need for resuming sedation.
\end{abstract}

Trial registration: Clinicaltrials.gov, NCT01193816. Registered on 26 August 2010.

Keywords: Mechanical ventilation, Ventilation weaning, Agitation, Intensive care unit

\section{Background}

Agitation is a frequent complication of critical illness, occurring in up to $50 \%$ of mechanically ventilated patients in the medical-surgical intensive care unit (ICU) [1]. It is associated with severe adverse events, including increased odds of unplanned extubation and central venous catheter removal, more frequent nosocomial infections and an increased duration of ICU stay [1]. Some preventive measures, including systematic evaluation of pain and agitation, have proven beneficial in reducing the incidence of agitation in the ICU [2]. Other

\footnotetext{
* Correspondence: didier.dreyfuss@lmr.aphp.fr; didier.dreyfuss@aphp.fr †Equal contributors

${ }^{1}$ Service de Réanimation Médico-Chirurgicale, Hôpital Louis Mourier, Assistance Publique - Hôpitaux de Paris, F-92700 Colombes, France ${ }^{8}$ Infection, Antimicrobials, Modelling, Evolution (IAME), UMR 1137, Université Paris Diderot, Sorbonne Paris Cité, F-75018 Paris, France

Full list of author information is available at the end of the article
}

measures, such as the use of sedation protocols to hasten weaning from mechanical ventilation (MV), may be associated with an increase in the incidence of agitation episodes [3, 4]. Current guidelines do not recommend the use of haloperidol or other typical antipsychotics to treat delirium and/or agitation [5]. Moreover, data on the safety of antipsychotics in the ICU setting and their efficacy to control agitation and improve patient outcomes are scarce. A randomised, double-blind, placebocontrolled trial of intravenous haloperidol in critically ill patients showed no effect on delirium incidence or duration. A secondary data analysis showed that the proportion of patients with a Richmond AgitationSedation Scale (RASS) score $\geq 2$ was lower in the haloperidol group than in the placebo group $(13 \%$ vs $20 \%$, $p=0.0075$ ), suggesting that it could be used for short-term 
management of acute agitation, despite having no effect on delirium [6].

Loxapine is a typical antipsychotic drug. A plasmatic peak is reached roughly $1.5 \mathrm{~h}$ after oral intake, and its half-life is around $8 \mathrm{~h}$. Side effects include drowsiness, extrapyramidal symptoms, tachycardia and hypotension. Its approved indications in France are acute and/or chronic psychotic states and severe agitation. It has been used for a long time, mainly in France, because of its efficacy, safety profile, availability and cost. During a physiological study, loxapine was found to be safe and effective in treating agitation in mechanically ventilated patients and accounted for improved respiratory parameters [7]. In this study, we aimed to test whether the use of loxapine in agitated patients during weaning from $\mathrm{MV}$ could reduce the duration of MV.

\section{Methods}

\section{Study design}

This prospective, randomised, double-blind, multicentre study was conducted over a 2-year period (November 2011 to November 2013) in five university-affiliated French ICUs. Written informed consent was obtained from patients' next of kin at inclusion in the study. In the absence of next of kin, informed consent could be waived, as allowed by the institutional review board according to French law that allows inclusion of patients in emergency conditions. Whatever the type of inclusion, all surviving patients were informed about the trial at the earliest opportunity after neurological recovery, and consent to continue in the trial was sought. An investigator at each centre was responsible for enrolling patients in the trial, following the protocol and completing the case report form. Two clinical research technicians regularly monitored the data.

The protocol was approved by an institutional review board, the Comité de Protection des Personnes Paris Ile de France I, according to French law. The study received no commercial support. This study was granted permission by the French Ministry of Health (PHRC P070106) and registered with ClinicalTrials.gov under the identifier NCT01193816.

\section{Participants}

Adult patients under $\mathrm{MV}$ for $>48 \mathrm{~h}$, meeting readiness-to-wean criteria (see below) and exhibiting agitation, as defined by a RASS score $\geq 2$ [8], at the time of sedation decrease or withdrawal were eligible and included in the absence of non-inclusion criteria. Non-inclusion criteria included pregnancy, contraindication to loxapine (e.g., hypersensitivity or known history of epilepsy), on-going treatment with a dopamine agonist, limitation of life-sustaining therapy, planned extubation in the following $24 \mathrm{~h}$, and contra- indication to enteral access through a nasogastric tube.

\section{Randomisation and masking}

Patients were randomised to receive loxapine or placebo immediately after inclusion. Study drug packs were prepared by the Louis Mourier Hospital Pharmacy Department (Colombes, France). The loxapine and placebo drug components of solution were packaged identically into numbered treatment packs matching the patients' study numbers. Each treatment pack contained 14 flasks of $60 \mathrm{ml}$. Each flask contained either $1.5 \mathrm{~g}$ of loxapine or placebo used for the scheduled administrations over a 24-h period. Care providers and investigators were blinded to group assignment.

\section{Sedation management}

In all participating centres, sedation consisted of continuous infusion of sufentanil and midazolam or propofol. Three centres used a written, nurse-implemented sedation protocol to reach an estimated appropriate depth of sedation on the basis of the RASS score. No written sedation protocol was used in the two other centres, and daily interruption of sedation was usual practice.

\section{Mechanical ventilation weaning}

Readiness to wean was assessed in daily screening. The criteria were defined according to usual guidelines [9]: (1) recovery from acute disease (e.g., pneumonia, chronic obstructive pulmonary disease exacerbation, extra-pulmonary infection, shock); (2) marked improvement of the reason for MV initiation; (3) haemodynamic stability without catecholamine infusion or a small and decreasing infusion rate; and (4) peripheral capillary oxygen saturation $\geq 92 \%$, fraction of inspired oxygen $\leq 50 \%$, positive end-expiratory pressure $\leq 5 \mathrm{cmH}_{2} \mathrm{O}$ and no sign of respiratory failure. Standard weaning from MV procedure was performed in all investigating centres [10]. The weaning test consisted of a T-tube trial with supplemental oxygen administration at four centres, and a pressure support trial with an inspiratory pressure of 5 $\mathrm{cmH}_{2} \mathrm{O}$ at one centre. The tube cuff was systematically kept inflated during T-tube trials.

Failure criteria encompassed respiratory rate $>35 / \mathrm{mi}-$ nute, arterial oxygen saturation $<90 \%$, heart rate $>140$ beats per minute or sustained increase or decrease in heart rate $>20 \%$, systolic blood pressure $>180 \mathrm{mmHg}$ or $<90 \mathrm{mmHg}$, agitation, diaphoresis or anxiety. Arterial blood gas analyses could be performed but were not mandatory.

In case of weaning trial success, extubation was performed if physiotherapy evaluation revealed appropriate cough and muscular strength. Arterial blood gas analyses were not systematically performed. If respiratory acidosis 
(hypercapnia $>45 \mathrm{mmHg}$ and $\mathrm{pH}<7.35$ ) was documented, the extubation could be delayed.

After extubation, patients received oxygen by mask or nasal cannula. Prophylactic non-invasive MV was not considered an exclusion criterion, because it can facilitate extubation of patients with chronic cardiac or respiratory impairment [11].

\section{Procedures}

During the study, no administration of a neuroleptic other than the study drug was allowed (Fig. 1).

\section{In case of RASS score of 2 at randomisation}

After randomisation, patients received $150 \mathrm{mg}$ of loxapine or placebo by nasogastric tube. Our group has previously found a time of $62 \pm 39$ minutes between loxapine enteral administration and effect [7]. Therefore, to avoid agitation-related side effects such as unplanned tube or vascular line removal, patients received at the same time a short administration of a low dose of benzodiazepine and/or morphinic agents in order to give time for the effect of loxapine. RASS score was monitored after 30, 60 and 90 minutes as well as $4 \mathrm{~h}$. A second dose of loxapine or placebo was administered between 90 minutes and $4 \mathrm{~h}$ after the first one in case of a persistent RASS score of 2 .

Then, RASS score was monitored every $4 \mathrm{~h}$ until $48 \mathrm{~h}$ after extubation (or up to 14 days in the absence of MV weaning). This monitoring allowed us to adapt study treatment as follows: RASS score $<1$, no loxapine or placebo re-administration; RASS score of 1 , administration of $50 \mathrm{mg}$ of loxapine or placebo; and RASS score of 2, administration of $50-100 \mathrm{mg}$ of loxapine or placebo (at the discretion of the attending intensivist).

If the RASS score was found to be $>2$ after randomisation, usual sedation (benzodiazepine and morphinic agents)

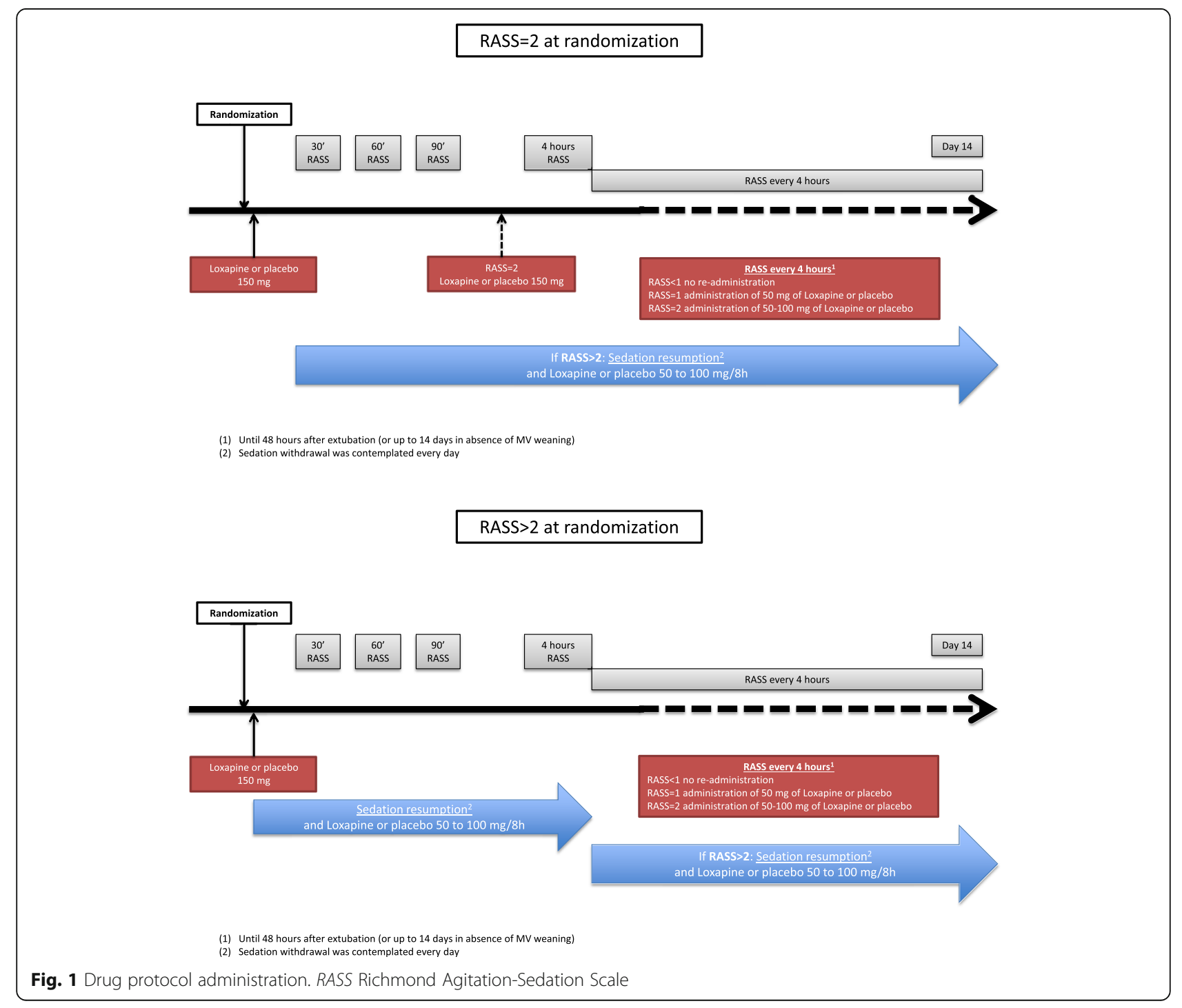


was immediately resumed. Thereafter, $100 \mathrm{mg}$ of loxapine or placebo was administered every $8 \mathrm{~h}$, and sedation withdrawal was contemplated every day. When this allowed sedation withdrawal, loxapine administration was continued according to the modalities explained above (depending on RASS score).

\section{In case of RASS score $>2$ at randomisation}

After randomisation, patients received $150 \mathrm{mg}$ of loxapine or placebo by nasogastric tube, and usual sedation (benzodiazepine and morphinic agents) was resumed at the same time. Then, $100 \mathrm{mg}$ of loxapine or placebo was administered every $8 \mathrm{~h}$, and sedation withdrawal was contemplated every day. When this allowed sedation withdrawal, loxapine administration was continued according to the modalities explained above (depending on RASS score).

Within $48 \mathrm{~h}$ following extubation, administration of loxapine or placebo was pursued, depending on RASS score: no administration when the RASS score was $<1$; administration of $0-50 \mathrm{mg}$ (at the discretion of the intensivist) when the RASS score was 1; and administration of $50-100 \mathrm{mg}$ (at the discretion of the attending intensivist) when the RASS score was 2 . In case of RASS score $>2$, control of agitation could include administration of a non-neuroleptic agent according to unit procedure.

\section{Data collection}

At the time of inclusion, we recorded data on age, sex, Simplified Acute Physiology Score II (SAPS II) [12], indication for MV, co-morbid conditions, alcohol consumption, toxic drug abuse and psychoactive drug use. Amounts of sedative agents given in the previous $24 \mathrm{~h}$ were registered. The Sepsis-related Organ Failure assessment score [13] was recorded at the time of randomisation. Both RASS score and the following physiological variables were monitored at 30, 60 and 90 minutes as well as $4 \mathrm{~h}$ after inclusion: temperature, respiratory rate, heart rate, systolic arterial pressure and airway occlusion pressure during the first 0.1 second of inspiration (P0.1). Then, RASS score, need for repeat loxapine or placebo administration and sedation resumption were recorded every $4 \mathrm{~h}$ until $48 \mathrm{~h}$ after extubation or until day 14. Indication for sedation resumption, sedation duration and nosocomial pneumonia occurrence were also recorded. Vital status was monitored 6 weeks after randomisation. The patients were closely screened for the following loxapine side effects: dyskinesia, seizure and unexplained fever.

\section{Outcome}

\section{Primary outcome}

The primary endpoint was the time between inclusion and successful extubation, as defined by the absence of re-intubation within $48 \mathrm{~h}$ after extubation.

\section{Secondary outcomes}

Secondary outcomes were the rate of unplanned extubation, ventilator-free days during the first 14 days, evolution of physiological parameters (in the first $4 \mathrm{~h}$ after randomisation), rate of sedation resumption in the first $24 \mathrm{~h}$ (this outcome has not been pre-specified on ClinicalTrials.gov), rate of nosocomial pneumonia, rate of loxapine side effects, mortality at 14 days and 6 weeks, and factors associated with unsuccessful weaning.

\section{Statistical analysis}

Sample size was determined on the basis of previously reported data $[10,14]$ indicating that median duration of weaning from MV ranges between 3 and 9 days. With a median duration of 6 days in the placebo group and a followup period of 14 days, 115 patients per group were required to demonstrate a decrease of 2 days of median duration of MV (primary endpoint) in the loxapine group, with an alpha risk of $5 \%$ and a power of $80 \%$. To take into account a death rate before successful extubation of about $25 \%, 150$ patients per group were planned (300 patients overall).

All analyses were based on the intention-to-treat principle. Baseline characteristics were described by group (placebo, loxapine) using frequency and percent for categorical variables and mean and SD for continuous variables (except for continuous variables with a non-normal distribution, for which we used median and IQR). The primary outcome was represented using the Kaplan-Meier method. Time to successful extubation was calculated from the time of inclusion of the patient. Deaths before any successful extubation were censored at the time of death. Patients were censored after 14 days if no event (extubation or death) occurred during the follow-up. The primary outcome was compared between the two groups with a log-rank test.

For secondary endpoints, the chi-square test or Fisher's exact test (as appropriate) was used to compare categorical variables, and Student's $t$ test or the Wilcoxon test (as appropriate) was used for continuous variables. Evolution of physiological (arterial pressure, heart rate, respiratory rate and temperature) and clinical (RASS score $\geq 2$ or $<2$ ) parameters during the first $4 \mathrm{~h}$ were compared between the placebo and loxapine groups with generalised estimating equation models in order to take repeated measurement of participants' responses into account, and thus the expected correlation within each participant. An identity link function was used for continuous parameters, and a logit link function was used for binary parameters. An unstructured correlation structure was used in all models.

\section{Role of the funding source}

The sponsor of the study (French Ministry of Health) had no role in study design, data collection, data 
analysis, data interpretation or writing of the report. SG, DH and DD had access to the raw data. The corresponding author (DD) had full access to all data and final responsibility for the decision to submit the report for publication.

\section{Results}

\section{Baseline characteristics}

From November 2011 through November 2013, a total of 1480 patients under MV for more than $48 \mathrm{~h}$ and meeting readiness-to-wean criteria were present in the participating ICUs (Fig. 2). The trial was discontinued after 102 patients had been randomised because of an insufficient rate of enrolment. Fifteen patients were excluded owing to absence or withdrawal of informed consent, leaving 87 patients for analysis: 48 in the loxapine group and 39 in the placebo group.

Except for age, the characteristics of the patients at inclusion were similar in the two groups, especially in terms of sedation dose in the $24 \mathrm{~h}$ before randomisation (Table 1).

The median time from ICU admission to study inclusion did not differ significantly between the loxapine group (5.5 [3.49-7.78] days) and the placebo group (5.66 [4.49-6.81] days) $(p=0.47)$.

\section{Outcomes}

\section{Primary outcome}

During the 14-day period from randomisation, the median times to successful extubation were 3.2 days in the loxapine group and 5 days in the placebo group (relative risk $1.2,95 \% \mathrm{CI} 0.75-1.88, p=0.45$ ). Figure 3 shows the daily rate of successful extubation in the loxapine and placebo groups until day 14. Three patients died within the 14 days of follow-up (one in the loxapine group, two in the placebo group), and only one death (placebo group) occurred when a patient was still under MV.

To investigate the intersecting appearance of the two curves, we performed a supplementary data analysis of the first $24 \mathrm{~h}$ after randomisation. This analysis revealed that six patients were extubated in the placebo group (including four unplanned extubations) and two were extubated in the loxapine group (planned extubations), accounting for a higher initial rate of extubation in the placebo group. The results of the primary outcome analysis after 14 days for a median follow-up of 29.5 days did not differ (data not shown).

\section{Secondary outcome}

Sedation was more frequently resumed in the placebo group ( $44 \%$ vs $17 \%, p=0.01$ ) during the first $24 \mathrm{~h}$ (Table 2 ), and the proportion of patients with RASS scores $\geq 2$ was significantly lower in the loxapine group $(p=0.003)$ during the $4 \mathrm{~h}$ following study drug administration (Fig. 4).

We identified no difference in either ventilator-free days during the first 14 days or rate of unplanned extubation, nosocomial pneumonia or mortality after 14 days and 6 weeks (Table 2). Total dose administered within the first $24 \mathrm{~h}$ did not differ between groups $(2[2,3]$ vs 3 [2-4], $p=0.22$ ). However, the total dose administered within the first $48 \mathrm{~h}$ and within 14 days were higher in the placebo group (4 [2-5] vs $5[4-6], p=0.03$; and 4 [3-7] vs 8 [4-14], $p=0.01)$.

The evolution of physiological parameters (arterial pressure, heart rate, respiratory rate and temperature) was not different during the first $4 \mathrm{~h}$ after randomisation (Fig. 5).

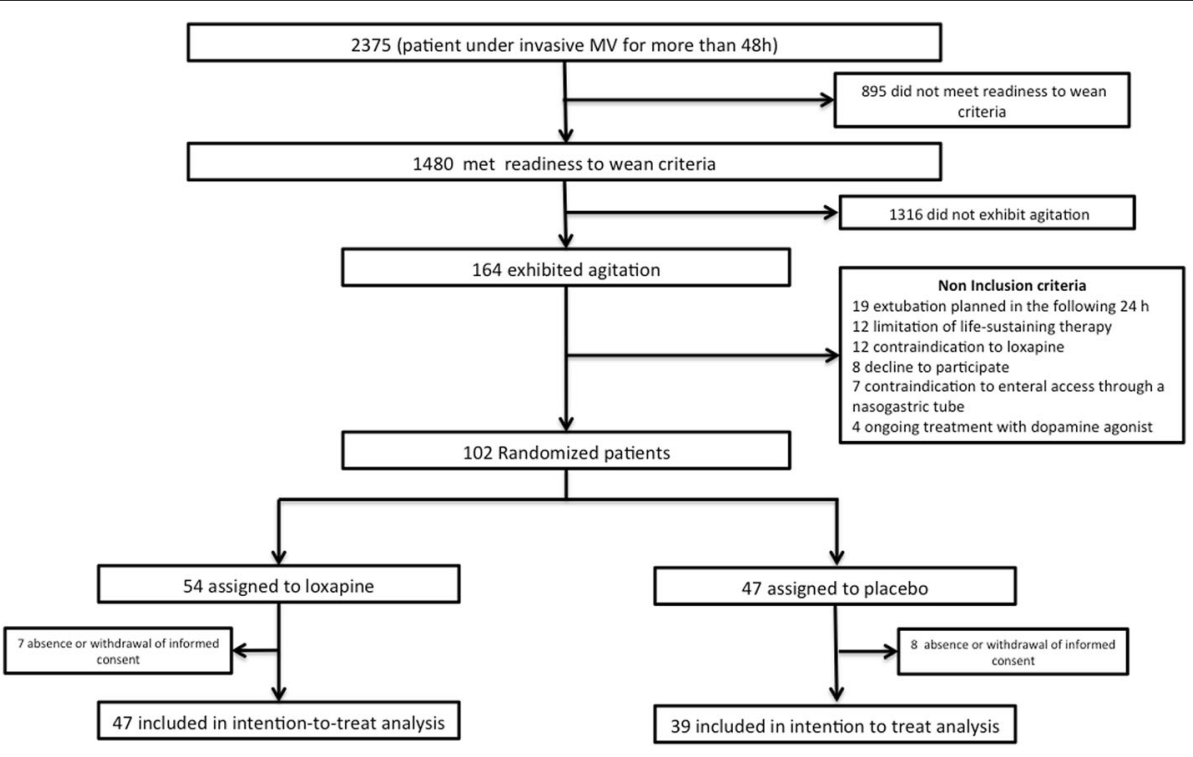

Fig. 2 Patients flowchart of the 2-year study period 
Table 1 Baseline characteristics of the intention-to-treat population

\begin{tabular}{|c|c|c|c|}
\hline & Loxapine $(n=48)$ & Placebo $(n=39)$ & $p$ Value \\
\hline Age, years (SD) & $59.6(15.6)$ & $51(17.7)$ & 0.02 \\
\hline Male sex, $n(\%)$ & $35(74.5)$ & $31(79.5)$ & 0.6 \\
\hline SAPS II score (SD) & $48.2(17.2)$ & $53.7(17.9)$ & 0.15 \\
\hline SOFA score [IQR] & $9[5-12]$ & $9[7-11]$ & 0.8 \\
\hline Alcohol consumption, n (\%) & $13(28)$ & $17(44)$ & 0.12 \\
\hline Toxic drug abuse, $n(\%)$ & $5(11)$ & $6(15)$ & 0.54 \\
\hline Psychoactive drug use, $n$ (\%) & $8(17)$ & $10(26)$ & 0.33 \\
\hline \multicolumn{4}{|l|}{ Indication for MV } \\
\hline Acute respiratory failure, $n(\%)$ & $23(49)$ & $16(41)$ & 0.46 \\
\hline Shock, $n(\%)$ & $12(25)$ & $9(23)$ & 0.48 \\
\hline Coma, n (\%) & $8(17)$ & $9(23)$ & 0.48 \\
\hline \multicolumn{4}{|l|}{ Co-morbid conditions } \\
\hline COPD, $n(\%)$ & $5(11)$ & $4(10)$ & 1.0 \\
\hline Congestive heart disease, $n(\%)$ & $6(13)$ & $4(10)$ & 1.0 \\
\hline Chronic renal failure, $n(\%)$ & $2(4)$ & $0(0)$ & 0.5 \\
\hline Hepatic disease, $n(\%)$ & $1(2)$ & $1(3)$ & 1.0 \\
\hline Duration of MV before randomisation, days [IQR] & $5[3-7]$ & $6[5-8]$ & 0.06 \\
\hline \multicolumn{4}{|c|}{ Cumulative amount of sedative drugs in the previous $24 \mathrm{~h}$} \\
\hline Midazolam, mg & $114[40-230]$ & $120[57-341]$ & 0.5 \\
\hline Fentanyl, $\mu \mathrm{g}$ & $2150[1731-4000]$ & $2700[2550-4300]$ & 0.6 \\
\hline Sufentanil, $\mu \mathrm{g}$ & $247.5[107-333.75]$ & $270[130-638.75]$ & 0.2 \\
\hline Propofol, mg & 1440 [750-2550] & 1520 [1085-2400] & 0.3 \\
\hline
\end{tabular}

Abbreviations: COPD Chronic obstructive pulmonary disease, MV Mechanical ventilation, SAPS II Simplified Acute Physiology Score II (calculated in the first $24 \mathrm{~h}$ of admission in ICU), SOFA Sepsis-related Organ Failure assessment (calculated at randomisation)

Data are $n(\%)$, median (IQR) or mean (SD)

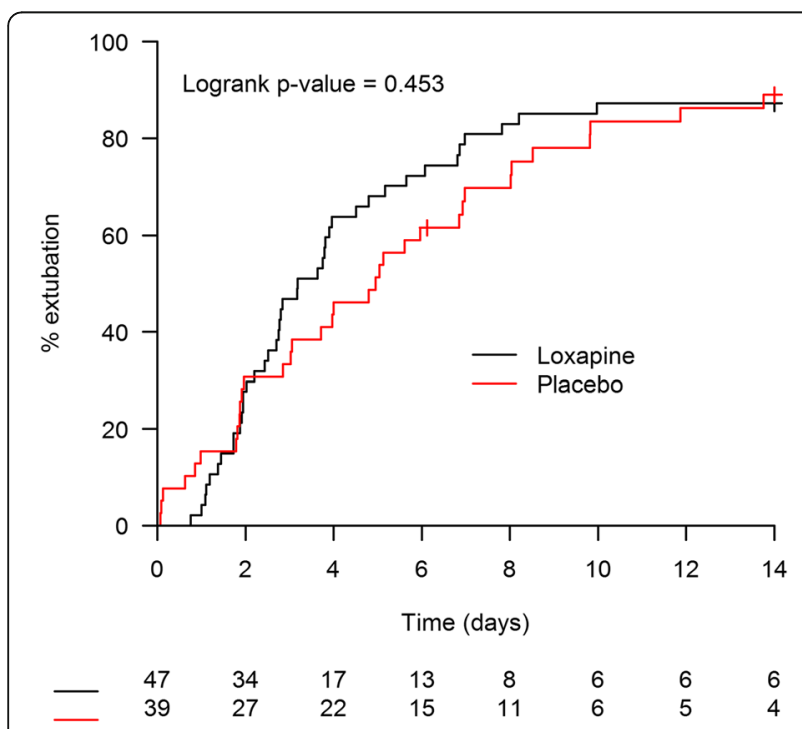

Fig. 3 Kaplan-Meier plot. Proportion of successful extubation during the 14 days following randomisation
Owing to a substantial amount of missing data, P0.1 was not analysed. Univariate analysis did not identify any factor associated with unsuccessful weaning 5 days after randomisation, particularly with regard to the randomisation group.

The rate of adverse events did not differ between the two groups (Table 3). One patient in the loxapine group had a transient seizure episode.

\section{Discussion}

In this prematurely stopped study of the effects of loxapine in agitated patients during weaning from invasive

Table 2 Secondary outcomes

\begin{tabular}{lllc}
\hline & Loxapine & Placebo & $p$ Value \\
\hline Ventilator-free days in first 14 days (SD) & $5.8(8.4)$ & $5.5(8.4)$ & 0.9 \\
Unplanned extubations, $n(\%)$ & $6(13)$ & $7(18)$ & 0.5 \\
Sedation resumption during the first & $8(17)$ & $17(44)$ & 0.01 \\
$24 \mathrm{~h}, n(\%)$ & & & \\
Nosocomial pneumonia, $n(\%)$ & $7(15)$ & $8(21)$ & 0.5 \\
Mortality at 14 days, $n(\%)$ & $1(2)$ & $2(5)$ & 0.6 \\
Mortality at 6 weeks, $n(\%)$ & $5(10)$ & $3(8)$ & 0.7 \\
\hline
\end{tabular}




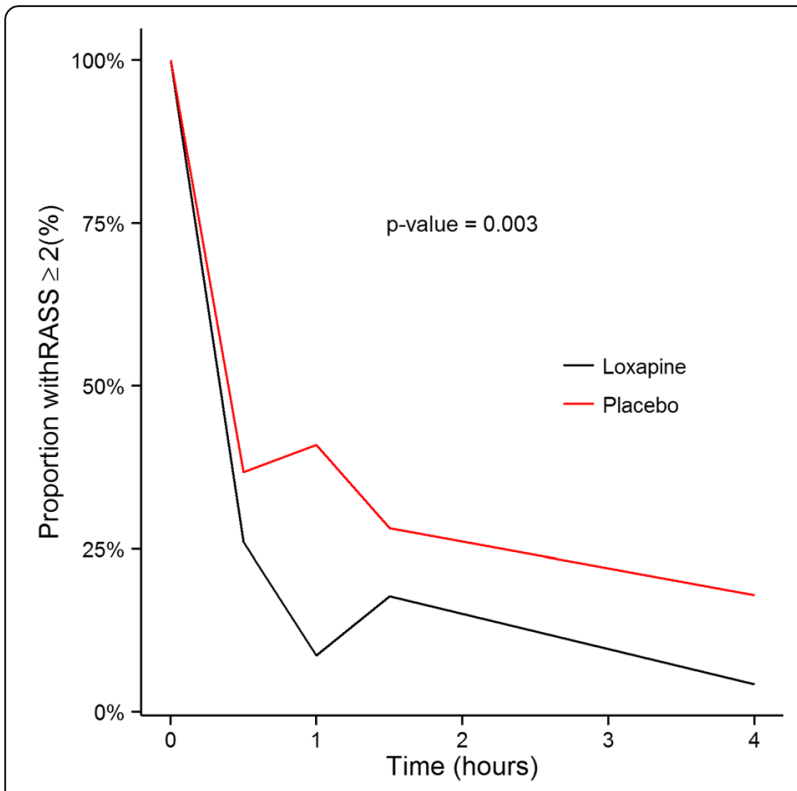

Fig. 4 Proportion of patients with agitation (Richmond AgitationSedation Scale [RASS] score $\geq 2$ ) during the first $4 \mathrm{~h}$ following randomisation. Sedation resumption may explain the RASS score reduction in placebo group. However, this reduction was more frequent in the loxapine group

MV, loxapine did not significantly shorten weaning from MV. However, loxapine administration significantly reduced the RASS score in the $4 \mathrm{~h}$ after randomisation and also reduced the need for sedation resumption in the subsequent $24 \mathrm{~h}$.

We hypothesised that loxapine might allow for a decrease of median duration of MV of 2 days. This hypothesis might have been correct because we observed median times from randomisation to successful extubation of 5 days in the placebo group and of 3.2 days in the loxapine group. Unfortunately, we randomised only 102 patients instead of the 300 initially scheduled, and analysis was possible in only 87 of them (because of absence or withdrawal of consent in 15), which precludes any conclusion based on the primary hypothesis. With the observed sample size, this study has a power of $40 \%$ to demonstrate a decrease of 2 days of median duration of $\mathrm{MV}$ in the loxapine group and a power of $80 \%$ to demonstrate a decrease of 3 days of median duration of MV in the loxapine group.

Lack of power might potentially explain the absence of difference in the primary outcome. Difficulties in recruiting patients were a consequence of the infrequency of isolated agitation during MV weaning, as attested by the flowchart. Several causes may explain these difficulties. First, use of sedative drugs has decreased in the ICU in recent years [15] as a probable consequence of major publications that promote less sedation $[3,16]$. In our study, participating centres used a written sedation protocol or daily sedation interruption [16] that could lead to less profound sedation and therefore less agitation during awakening. Second, participating centres took great care in conducting pain evaluation and management, which may also have resulted in less frequent agitation occurrence. Third, the non-inclusion criterion of extubation planned in the following $24 \mathrm{~h}$ could be too subjective. Indeed, during the screening period, 19 patients were not included because of this criterion.

This study is the first double-blind, placebo-controlled, randomised trial in which loxapine for agitation was tested during weaning from invasive MV. We found that loxapine significantly reduced agitation, as attested by RASS score decrease in the first $4 \mathrm{~h}$ after randomisation, without significant adverse outcomes. Sedative drug administration (increase of dosage or resumption) is often required to control agitation during weaning from invasive $\mathrm{MV}$ [17]. In our study, sedation resumption was needed significantly less often with loxapine than with placebo. This result is an important strength of this study because prolonged sedation has several drawbacks, including limitation of clinicians' ability to interpret physical examinations; promotion of delirium [18]; and prolongation of invasive MV, ICU stay and hospital stay $[3,19]$.

The intersecting appearance of the two curves shown in Fig. 2 is explained by more unplanned extubation in the placebo group during the $24 \mathrm{~h}$ following randomisation (four unplanned extubations in the placebo group vs none in the loxapine group). Unplanned extubation may be associated with serious complications [20]. Although the effects of unplanned extubation on nosocomial pneumonia are debated [21-23], unplanned extubation seems to prolong ICU stay [24], and it is an indicator of quality of care in the ICU $[23,25]$.

Our trial has potential limitations. First, only three centres used written protocols for sedation. However, a significant influence of sedation management on the primary outcome is unlikely because it is a double-blind, placebo-controlled trial. Second, the use of non-invasive ventilation to treat post-extubation respiratory failure may create heterogeneity in the primary endpoint. Unfortunately, data on non-invasive respiratory support (non-invasive ventilation, high-flow oxygen nasal cannula) were not collected. These heterogeneities in practice may constitute confounding factors. However, such confounding is very unlikely to affect the results. Indeed, if it had any effect, it would likely be to decrease the capacity of proving a difference (type II error). Our study was stopped prematurely after the inclusion of one-third of the expected population. This constitutes the most plausible explanation for lack of significance. The effect of confounding, if any, would be to further decrease the significance of the results. On the contrary, it seems more than improbable that confounders would falsely 

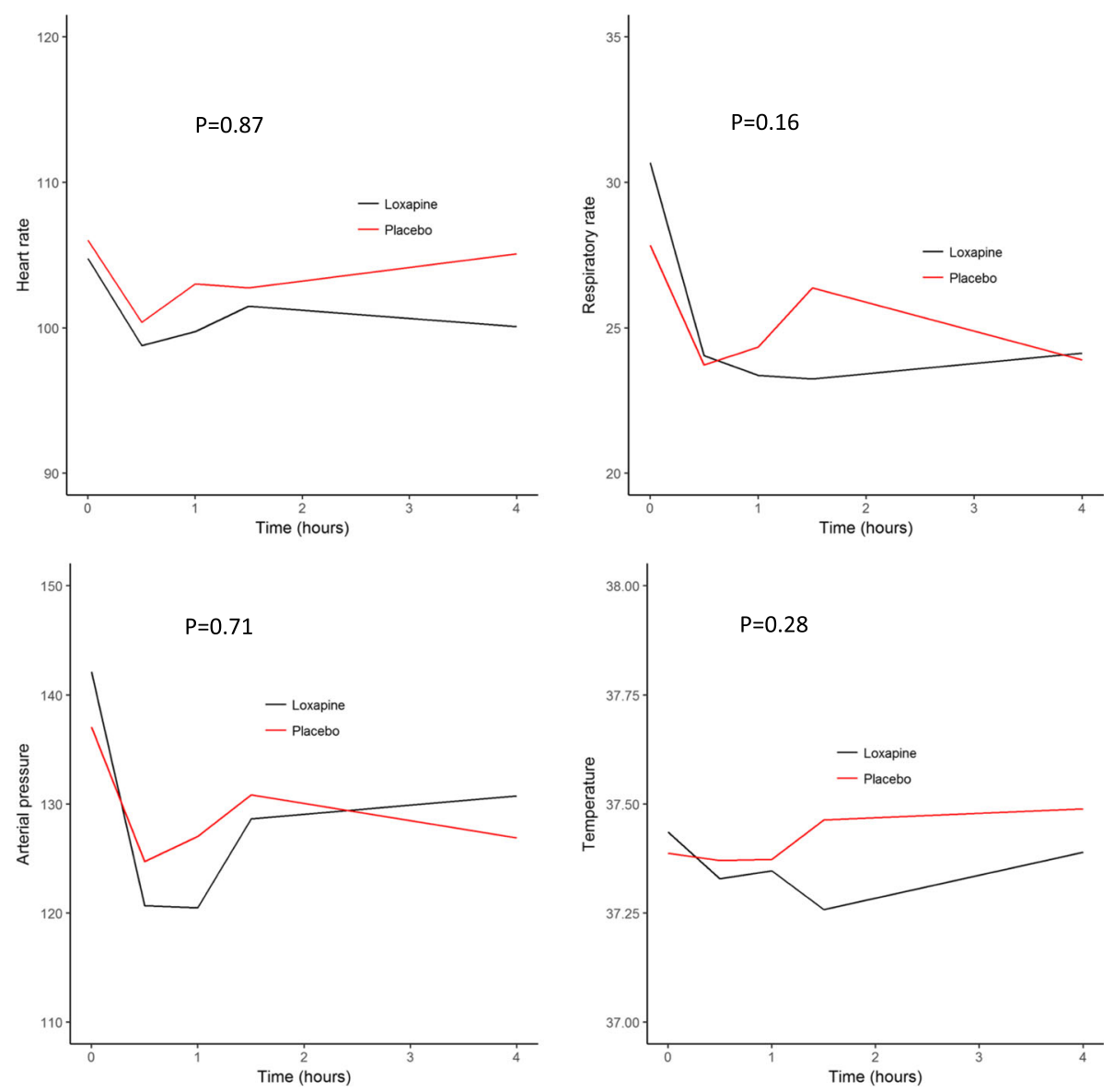

Fig. 5 Evolution of physiological parameters (arterial pressure, heart rate, respiratory rate and temperature) during the first $4 \mathrm{~h}$ after randomisation

increase the probability of finding a significant difference (type I error) because our results are negative. In any case, a larger-scale randomised controlled trial is mandatory to evaluate the potential of loxapine in this context and should minimise heterogeneity, taking the above-mentioned confounding factors into account.

The dose of loxapine in the protocol followed the industry instruction and was validated by our hospital pharmacist. Antipsychotic agents have been tested more often to prevent or treat delirium in the ICU [6, 26-29] than for agitation during weaning from MV. A pilot randomised, placebo-controlled study including 36 patients showed that quetiapine added to as-needed haloperidol resulted in faster delirium resolution and less agitation [27]. No larger randomised trial was performed to

Table 3 Main adverse events

\begin{tabular}{llll}
\hline & Loxapine & Placebo & $p$ Value \\
\hline Shock, $n(\%)$ & $5(10)$ & $6(15)$ & 0.54 \\
Unexplained fever, $n(\%)$ & $1(2)$ & $0(0)$ & 1.0 \\
Seizure, $n(\%)$ & $1(2)$ & $0(0)$ & 1.0 \\
Dyskinesia, $n(\%)$ & 0 & 0 & \\
\hline
\end{tabular}

confirm these results. More recently, in a randomised, double-blind, placebo-controlled trial including 142 patients, intravenous haloperidol did not modify the duration of delirium in critically ill patients [6]. In this trial, patients receiving haloperidol were less frequently agitated, however, leading the authors to conclude that the use of haloperidol should be reserved for short-term management of acute agitation. Haloperidol has numerous drawbacks, including extra-pyramidal manifestations and significant QTc interval prolongation [30, 31]. Because of its good haemodynamic safety profile and the rarity of its side effects, loxapine has been used routinely in many ICUs in France. We observed a single episode of seizure in one patient in the loxapine group, but no other adverse effects. This seems to confirm the safety of use of this drug, but it emphasises the need for close patient monitoring. We previously showed that loxapine allowed for adequate control of agitation with normalisation of several physiological parameters during weaning from MV [7]. To our knowledge, these potentially interesting properties have not previously been tested in a randomised controlled trial. In addition, there is a lack of data on the dose of loxapine which is required to 
control agitation in the ICU. Our study suggests that a first administration of $150 \mathrm{mg}$ and then a maintenance dose of $50-100 \mathrm{mg}$ every 4 or $8 \mathrm{~h}$ in case of persistent agitation is safe and efficient for treating acute agitation.

\section{Conclusions}

Loxapine allowed agitation control during MV weaning and decreased the need for sedation resumption compared with placebo. However, loxapine did not significantly shorten weaning from MV. Altogether, our study constitutes a firm rationale for undertaking a more powered one to assess the potential benefit of loxapine in agitated patients during weaning from MV.

\section{Abbreviations \\ COPD: Chronic obstructive pulmonary disease; ICU: Intensive care unit; MV: Mechanical ventilation; RASS: Richmond Agitation-Sedation Scale; SAPS II: Simplified Acute Physiology Score II; SOFA: Sepsis-related Organ Failure assessment}

\section{Acknowledgements}

We thank Clémentine Rappaport, MD, for her suggestion to use loxapine for management of agitation in daily ICU practice. We thank the pharmacy department of Louis Mourier Hospital (Colombes, France) for the study drug pack preparations.

\section{Funding}

The work was supported by the French Ministry of Health (PHRC P070106).

\section{Availability of data and materials}

The datasets used and analysed during the present study are available from the corresponding author on reasonable request.

\section{Authors' contributions}

DD, SG, BS and LS were responsible for study conception and contributed to study design and analysis as well as interpretation of data and obtaining funding. SG, DD, BS and JDR were responsible for drafting of the manuscript. $\mathrm{DH}$ contributed to statistical analysis and interpretation of data. BM, GVDM, $D V, Y C$ and RS contributed to acquisition of data and drafting of the manuscript. All authors read, reviewed and approved the final manuscript.

\section{Ethics approval and consent to participate}

The protocol was approved by an institutional review board, the Comité de Protection des Personnes Paris lle de France I, according to French law. Written informed consent was obtained from patients' next of kin at the time of inclusion in the study. In the absence of next of kin, informed consent could be waived as allowed by the institutional review board according to French law that allows inclusion of patients in emergency conditions. Whatever the type of inclusion, all surviving patients were informed about the trial at the earliest opportunity after neurological recovery, and consent to continue in the trial was sought. Among 102 randomised patients, 15 patients were excluded owing to absence or withdrawal of informed consent.

\section{Consent for publication}

Not applicable.

\section{Competing interests}

The authors declare that they have no competing interests

\section{Publisher's Note}

Springer Nature remains neutral with regard to jurisdictional claims in published maps and institutional affiliations.

\section{Author details}

'Service de Réanimation Médico-Chirurgicale, Hôpital Louis Mourier, Assistance Publique - Hôpitaux de Paris, F-92700 Colombes, France.
${ }^{2}$ Epidémiologie Clinique et Évaluation Économique Appliqué aux Populations Vulnérables (ECEVE), UMR 1123, Université Paris Diderot, Sorbonne Paris Cité, F-75018 Paris, France. ${ }^{3}$ Service de Réanimation Polyvalente et surveillance continue, Hôpital Antoine Béclère, Assistance Publique - Hôpitaux de Paris, F-92140 Clamart, France. ${ }^{4}$ INSERM U999, Université Paris Sud, F-92060 Le Plessis Robinson, France. ${ }^{5}$ Service de Réanimation Médicale et maladies infectieuses, Hôpital Bichat, Assistance Publique - Hôpitaux de Paris, Paris, France. ${ }^{6}$ Service de Réanimation Médicale, Hôpital Lariboisière, Assistance Publique - Hôpitaux de Paris, Paris, France. ${ }^{7}$ Service de Réanimation Médico-Chirurgicale, Hôpital Avicenne, Assistance Publique - Hôpitaux de Paris, Bobigny, France. ${ }^{8}$ Infection, Antimicrobials, Modelling, Evolution (IAME), UMR 1137, Université Paris Diderot, Sorbonne Paris Cité, F-75018 Paris, France. ${ }^{9}$ Département d'Epidémiologie et Recherche Clinique, Hôpital Louis Mourier, Assistance Publique - Hôpitaux de Paris, 178 rue des Renouillers, F-92700 Colombes, France. ${ }^{10}$ Present address: Intensive Care Unit, Hôpital Louis Mourier, 178 rue des Renouillers, 92110 Colombes, France.

Received: 27 February 2017 Accepted: 22 August 2017

Published online: 06 September 2017

\section{References}

1. Jaber S, Chanques G, Altairac C, Sebbane M, Vergne C, Perrigault PF, Eledjam JJ. A prospective study of agitation in a medical-surgical ICU: incidence, risk factors, and outcomes. Chest. 2005:128:2749-57.

2. Chanques $G$, Jaber S, Barbotte E, Violet S, Sebbane M, Perrigault PF, Mann C, Lefrant JY, Eledjam JJ. Impact of systematic evaluation of pain and agitation in an intensive care unit. Crit Care Med. 2006;34:1691-9.

3. Strøm T, Martinussen T, Toft P. A protocol of no sedation for critically ill patients receiving mechanical ventilation: a randomised trial. Lancet. 2010;375:475-80

4. Curley MAQ, Wypij D, Watson RS, Grant MJC, Asaro LA, Cheifetz IM, Dodson BL, Franck LS, Gedeit RG, Angus DC, Matthay MA, RESTORE Study Investigators and the Pediatric Acute Lung Injury and Sepsis Investigators Network. Protocolized sedation vs usual care in pediatric patients mechanically ventilated for acute respiratory failure: a randomized clinical trial. JAMA. 2015:313:379-89.

5. Barr J, Fraser GL, Puntillo K, Ely EW, Gélinas C, Dasta JF, Davidson JE, Devlin JW, Kress JP, Joffe AM, Coursin DB, Herr DL, Tung A, Robinson BRH, Fontaine DK, Ramsay MA, Riker RR, Sessler CN, Pun B, Skrobik Y, Jaeschke R, American College of Critical Care Medicine. Clinical practice guidelines for the management of pain, agitation, and delirium in adult patients in the intensive care unit. Crit Care Med. 2013;41:263-306.

6. Page VJ, Ely EW, Gates S, Zhao XB, Alce T, Shintani A, Jackson J, Perkins GD, McAuley DF. Effect of intravenous haloperidol on the duration of delirium and coma in critically ill patients (Hope-ICU): a randomised, double-blind, placebo-controlled trial. Lancet Respir Med. 2013;1:515-23.

7. Sztrymf B, Chevrel G, Bertrand F, Margetis D, Hurel D, Ricard J-D, Dreyfuss D. Beneficial effects of loxapine on agitation and breathing patterns during weaning from mechanical ventilation. Crit Care. 2010;14:R86.

8. Sessler CN, Gosnell MS, Grap MJ, Brophy GM, O'Neal PV, Keane KA, Tesoro EP, Elswick RK. The Richmond Agitation-Sedation Scale: validity and reliability in adult intensive care unit patients. Am J Respir Crit Care Med. 2002;166:1338-44.

9. Boles JM, Bion J, Connors A, Herridge M, Marsh B, Melot C, Pearl R, Silverman $\mathrm{H}$, Stanchina M, Vieillard-Baron A, Welte T. Weaning from mechanical ventilation. Eur Respir J. 2007;29:1033-56.

10. Esteban A, Frutos F, Tobin MJ, Alía I, Solsona JF, Valverdú I, Fernández R, de la Cal MA, Benito S, Tomás R. A comparison of four methods of weaning patients from mechanical ventilation. Spanish Lung Failure Collaborative Group. N Engl J Med. 1995;332:345-50.

11. Thille AW, Cortés-Puch I, Esteban A. Weaning from the ventilator and extubation in ICU. Curr Opin Crit Care. 2013;19:57-64.

12. Le Gall JR, Lemeshow S, Saulnier F. A new Simplified Acute Physiology Score (SAPS II) based on a European/North American multicenter study. JAMA. 1993;270:2957-63.

13. Vincent JL, Moreno R, Takala J, Willatts S, De Mendonça A, Bruining H, Reinhart CK, Suter PM, Thijs LG. The SOFA (Sepsis-related Organ Failure Assessment) score to describe organ dysfunction/failure. Intensive Care Med. 1996;22:707-10.

14. Brochard L, Rauss A, Benito S, Conti G, Mancebo J, Rekik N, Gasparetto A Lemaire F. Comparison of three methods of gradual withdrawal from ventilatory support during weaning from mechanical ventilation. Am J Respir Crit Care Med. 1994;150:896-903. 
15. Ranzani OT, Simpson ES, Augusto TB, Cappi SB, Noritomi DT, AMIL Critical Care Group. Evaluation of a minimal sedation protocol using ICU sedative consumption as a monitoring tool: a quality improvement multicenter project. Crit Care. 2014;18:580.

16. Kress JP, Pohlman AS, O'Connor MF, Hall JB. Daily interruption of sedative infusions in critically ill patients undergoing mechanical ventilation. N Engl J Med. 2000;342:1471-7.

17. Chevrolet JC, Jolliet P. Clinical review: agitation and delirium in the critically ill - significance and management. Crit Care. 2007;11:214.

18. Pandharipande P, Shintani A, Peterson J, Pun BT, Wilkinson GR, Dittus RS, Bernard GR, Ely EW. Lorazepam is an independent risk factor for transitioning to delirium in intensive care unit patients. Anesthesiology. 2006;104:21-6

19. Kollef MH, Levy NT, Ahrens TS, Schaiff R, Prentice D, Sherman G. The use of continuous i.v. sedation is associated with prolongation of mechanical ventilation. Chest. 1998;114:541-8.

20. Coppolo DP, May JJ. Self-extubations: a 12-month experience. Chest. 1990;98:165-9.

21. Zwillich CW, Pierson DJ, Creagh CE, Sutton FD, Schatz E, Petty TL. Complications of assisted ventilation: a prospective study of 354 consecutive episodes. Am J Med. 1974;57:161-70.

22. Boulain T, Association des Réanimateurs du Centre-Ouest. Unplanned extubations in the adult intensive care unit: a prospective multicenter study. Am J Respir Crit Care Med. 1998:157:1131-7.

23. De Lassence A, Alberti C, Azoulay E, Le Miere E, Cheval C, Vincent F, Cohen Y, Garrouste-Orgeas M, Adrie C, Troche G, Timsit JF, OUTCOMEREA Study Group. Impact of unplanned extubation and reintubation after weaning on nosocomial pneumonia risk in the intensive care unit: a prospective multicenter study. Anesthesiology. 2002;97:148-56.

24. Chuang ML, Lee CY, Chen YF, Huang SF, Lin IF. Revisiting unplanned endotracheal extubation and disease severity in intensive care units. PLoS One. 2015;10:e0139864

25. Peñuelas Ó, Frutos-Vivar F, Esteban A. Unplanned extubation in the ICU: a marker of quality assurance of mechanical ventilation. Crit Care. 2011;15:128.

26. Prakanrattana $\mathrm{U}$, Prapaitrakool $\mathrm{S}$. Efficacy of risperidone for prevention of postoperative delirium in cardiac surgery. Anaesth Intensive Care. 2007;35:714-9.

27. Devlin JW, Roberts RJ, Fong JJ, Skrobik Y, Riker RR, Hill NS, Robbins T, Garpestad E. Efficacy and safety of quetiapine in critically ill patients with delirium: a prospective, multicenter, randomized, double-blind, placebocontrolled pilot study. Crit Care Med. 2010;38:419-27.

28. Wang W, Li HL, Wang DX, Zhu X, Li SL, Yao GQ, Chen KS, Gu XE, Zhu SN Haloperidol prophylaxis decreases delirium incidence in elderly patients after noncardiac surgery: a randomized controlled trial. Crit Care Med. 2012;40:731-9.

29. Girard TD, Pandharipande PP, Carson SS, Schmidt GA, Wright PE, Canonico AE, Pun BT, Thompson JL, Shintani AK, Meltzer HY, Bernard GR, Dittus RS, Ely EW, MIND Trial Investigators. Feasibility, efficacy, and safety of antipsychotics for intensive care unit delirium: the MIND randomized, placebo-controlled trial. Crit Care Med. 2010;38:428-37.

30. Garcia E, Robert M, Peris F, Nakamura H, Sato N, Terazawa Y. The efficacy and safety of blonanserin compared with haloperidol in acute-phase schizophrenia: a randomized, double-blind, placebo-controlled, multicentre study. CNS Drugs. 2009;23:615-25.

31. Skrobik YK, Bergeron N, Dumont M, Gottfried SB. Olanzapine vs haloperidol: treating delirium in a critical care setting. Intensive Care Med. 2004;30:444-9.

\section{Submit your next manuscript to BioMed Central and we will help you at every step:}

- We accept pre-submission inquiries

- Our selector tool helps you to find the most relevant journal

- We provide round the clock customer support

- Convenient online submission

- Thorough peer review

- Inclusion in PubMed and all major indexing services

- Maximum visibility for your research

Submit your manuscript at www.biomedcentral.com/submit 